INPLASY

PROTOCOL

To cite: Fan et al. Relative efficacy and safety of topical and oral NSAIDs in the treatment of osteoarthritis: systematic review and metaanalysis. Inplasy protocol

2021110009. doi:

10.37766/inplasy2021.11.0009

Received: 03 November 2021

Published: 03 November 2021

Corresponding author:

Yu Hui Wang

wangyuhuitg2017@163.com

Author Affiliation:

GuiLin Medicine University

Support: Eight laurels team.

Review Stage at time of this submission: Formal screening of search results against eligibility criteria.

Conflicts of interest:

None declared.

\section{Relative efficacy and safety of topical and oral NSAIDs in the treatment of osteoarthritis: systematic review and meta-analysis}

Fan, $\mathrm{MZ}^{1}$; Wang, $\mathrm{YH}^{2}$.

Review question / Objective: The aim of this meta-analysis of randomized controlled trials is to found out the difference in efficacy and safety between them according to the results. In order to verify whether topic NSAIDs really have the same efficacy and higher safety compared with oral NSAIDs.

Condition being studied: Osteoarthritis (OA) is the most prevalent chronic joint disease. the clinical manifestations are degeneration of articular cartilage and secondary hyperosteogeny. It usually occurs in the hands, knees and hip joints, resulting in joint pain, physical stiffness and decline in quality of life. So far, there is still no cure for OA, but effective drugs and non-drug interventions can reduce the pain and improve the quality of life of OA patients. The Chinese OA diagnosis and treatment guidelines point out that the key to $O A$ treatment is to relieve pain and improve joint function. The management of $O A$ related pain includes lifestyle changes and the use of analgesics (such as acetaminophen) as well as topical and nutritional drugs.

INPLASY registration number: This protocol was registered with the International Platform of Registered Systematic Review and Meta-Analysis Protocols (INPLASY) on 03 November 2021 and was last updated on 03 November 2021 (registration number INPLASY2021110009).

\section{INTRODUCTION}

Review question / Objective: The aim of this meta-analysis of randomized controlled trials is to found out the difference in efficacy and safety between them according to the results. In order to verify whether topic NSAIDs really have the same efficacy and higher safety compared with oral NSAIDs.

Condition being studied: Osteoarthritis $(O A)$ is the most prevalent chronic joint disease. the clinical manifestations are degeneration of articular cartilage and secondary hyperosteogeny. It usually occurs in the hands, knees and hip joints, 
resulting in joint pain, physical stiffness and decline in quality of life. So far, there is still no cure for $O A$, but effective drugs and non-drug interventions can reduce the pain and improve the quality of life of $O A$ patients. The Chinese OA diagnosis and treatment guidelines point out that the key to OA treatment is to relieve pain and improve joint function. The management of $O A$ related pain includes lifestyle changes and the use of analgesics (such as acetaminophen) as well as topical and nutritional drugs.

\section{METHODS}

Participant or population: Randomized controlled trials that enrolled patients should fulfill the OA radiology criteria, who have a history of pain for at least one month and have morning stiffness for more than 30 minutes. The pain of the participants was in a moderate position on the pain score scale of osteoarthritis and needed to take non-steroidal antiinflammatory drugs or other painkillers. Patients included should not include pregnant women, people allergic to nonsteroidal anti-inflammatory drugs, people with cardiovascular diseases and skin diseases. In addition, patients with rheumatoid arthritis, psoriatic arthritis, septic arthritis, gout and other types of arthritis will also be excluded.

Intervention: All types of oral non-steroidal anti-inflammatory drugs, including tablets, capsules, solutions, etc.

Comparator: The dosage forms of topic medication include patch, solution and with DMSO as carrier.

Study designs to be included: This systematic review included only those RCTs that had been peer-reviewed. All RCTs comparing topic with oral treatment of $O A$ in the relevant database were included in this paper. Non randomized controlled trials, review reports, pathological reports and only summary or meeting reports were excluded.
Eligibility criteria: We will make a standard data collection sheet before data extraction. Two reviewers will independently extract data from the selected studies and fill in the data collection sheet. Discrepancies and uncertainties will be resolved by consensus between the 2 review authors by asking the third author to make a final decision. We will extract the following data: 1. General information: the first author, title, the journal, publication type, publication year, country; 2. Methods: study design, sample size, randomization, allocation concealment, blinding methods, inclusion criteria, and exclusion criteria; 3 . Participants: age, gender, baseline disease activity; 4. Interventions: type of control, duration of treatment, frequency of treatment; 5. Outcomes: primary and secondary outcomes, adverse effects, and follow up.

Information sources: We will search articles in four electronic database including PubMed, Embase, Cochrane Library and Web of Science. All the English publications until May 2021 will be searched without any restriction of countries or article type. Reference list of all selected articles will independently screened to identify additional studies left out in the initial search.

Main outcome(s): The main outcome measures of this review were the subjective perception of pain measured by the visual analogue scale (VAS) and the Osteoarthritis Index (WOMAC) of the universities of Western Ontario and McMaster.

Additional outcome(s): Secondary outcomes were functional improvement and the incidence of adverse events.

Quality assessment / Risk of bias analysis: Two review authors will independently use the criteria outlined in the Cochrane Handbook for Systematic Reviews of Interventions to assess the risk of bias in the included studies. The following 7 domains in the Cochrane "Risk of bias tool" will be assessed:1. random sequence 
generation; 2. allocation concealment; 3 . blinding of participants and personnel;4. blinding of outcome assessment; 5 . completeness of outcome data; 6.completeness of reporting; 7.other sources of bias. results of bias assessment will be presented in a figure and a graph indicating low, high or unclear risk of bias for each of the 6 items in each trial. Sensitivity analysis will be conducted based on the bias assessment to assess robustness of results

Strategy of data synthesis: We will use RevMan $\mathbf{5 . 3}$ for all statistical analysis. If considerable heterogeneity is observed, joint effect estimates are analyzed using a $95 \% \mathrm{Cl}$ stochastic effects model. If necessary, each subgroup will be analyzed carefully.

Subgroup analysis: There is no pregrouping plan. Subgroup analysis was performed based on control interventions and different outcomes. As there are few literatures to be included and there is no great difference, subgroup analysis is not carried out.

Sensitivity analysis: We will use RevMan for all statistical analysis. If considerable heterogeneity is observed, joint effect estimates are analyzed using a $95 \% \mathrm{Cl}$ stochastic effects model. If necessary, each subgroup will be analyzed carefully.

Language: English.

Country(ies) involved: China.

Keywords: osteoarthritis; topical nonsteroidal anti-inflammatory drugs; oral topical non-steroidal anti-inflammatory drugs.

Contributions of each author:

Author 1 - Miao Zhen Fan - Investigation, Methodology and Writing - original draft.

Author 2 - Yu Hui Wang - Conceptualization and Data curation. 